\title{
A novel optimization framework for integrated design and control using a dynamic inversely controlled process model
}

\author{
Mahdi Sharifzadeh ${ }^{\text {a,* }}$, Nina F. Thornhill ${ }^{\text {a }}$ \\ ${ }^{a}$ Centre for Process System Engineering (CPSE), Department of Chemical Engineering, Imperial College \\ London, \\ *Email: mahdi@imperial.ac.uk. Address: Department of Chemical Engineering, Imperial College London, \\ South Kensington Campus, London SW7 2AZ.
}

\begin{abstract}
The profitability of chemical processes depends on their design and control. In practice, however, the design and control are often done in sequence, leaving little room for improving the control performance when the process design is already fixed. Many commentators have suggested design and control should be integrated. The difficulty associated with the integrated approach, however, is that the number of alternative designs increases with the size of the process. This article proposes an optimization framework using an inversely controlled process model. The combinatorial complexities associated with the controllers are disentangled from the formulation, but the process and its control structure are still designed simultaneously. This article also considers the effect of uncertainties on the optimal design. The proposed optimization framework is benchmarked on a case study from the literature using stochastic mixed integer dynamic programming. The results demonstrate the advantages of the proposed optimization framework.
\end{abstract}

Keywords: integrated design and control of chemical processes, co-design, stochastic mixed integer dynamic optimization, control structure selection, optimization under uncertainty. 


\section{Introduction}

The current industrial practice for the design of chemical processes and their control systems is sequential in that the control system is designed after the process has been designed [1]. However, the design of a process and the design of its control system share important decisions. Luyben [2] recognized a conflict between steady-state economics and dynamic performance of chemical processes. He presented a list of examples in which steady-state optimization of chemical processes limits their dynamic performance and showed that a systematic framework is needed to establish a trade-off between steady-state economics and dynamic performance objectives.

The integrated design of a chemical process and its control system is known as co-design. In codesign, the structural and parametric decisions involved in both process design and control design, are decided simultaneously leading to economic benefits and improvements in the process performance [3]. Sakizlis, Perkins and Pistikopoulos [4] reviewed the optimization methods applied to co-design, and classified them based on their decision-making criteria which may require steady-state or dynamic analysis. The type of modelling technique (linear or nonlinear, steady state or dynamic) which should be used in the co-design framework and the type of information (controllability, robustness, operability) that can be extracted from such a model is ongoing research, [5-8]. Sakizlis, et al [4] concluded that future research must focus on application of control theory in the optimization formulation. Klatt and Marquardt [9] presented a recent overview of research directions in process system engineering. They concluded that problem formulations are getting more and more integrated and evolving from steady-state lumped models to dynamic and distributed models. They suggested that the integration of process design and control is a crucial and open issue "for process development and operation, both from a technical and an economic perspective”

The co-design problem has a combinatorial characteristic that can be mostly attributed to the design of controllers. The reason is that the design of the controllers needs decisions on the pairing of manipulated and controlled variables, the type of controller, and values for the controller parameters. For example, Moon, et al. [10] considered a two-level hierarchical optimization framework in which the controller design is addressed in another optimization level to make it more tractable.

Sharifzadeh and Thornhill [11] have presented an optimization framework using the assumption of perfect control. In the perfect control framework, an inverse steady-state process model replaces the combined model of the chemical process and its controllers. It solves the combinatorial problem by disentangling the design of the controllers from the problem formulation using a steady-state perfect control assumption. Since steady-state analysis considers only initial and ultimate states of the process, the approach of [11] ensures only state controllability of the process and ignores the dynamic (i.e. functional) controllability. This paper introduces a modelling approach termed inversely controlled process model which also ensures functional controllability. Although the complexities associated with the controllers are disentangled from the proposed co-design framework, still the process and its control structure are decided simultaneously and the interactions between process design and control design are fully captured.

A further concern is that an operating process will be subject to disturbances. Therefore the co-design optimization problem is treated as optimization under uncertainty.

A methodology for addressing co-design problem should have following properties:

1. The problem representation must include all alternative solutions.

2. A comparison criterion (objective function) must screen between alternative solutions.

3. The complexity of the problem representation should be manageable. Most optimization algorithms rely on gradient information and good starting points and are sensitive to unstable solutions in the problem formulation. 
4. The problem representation must consider the uncertainties in the problem. This can be done by considering the likelihoods of disturbances.

The co-design optimization framework presented in this article meets all these requirements by applying the concept of perfect control, in order to reduce the complexity of the co-design problem. This framework is developed in Section 2. by modifying the conventional co-design approach. The modification is based on the concept of functional controllability and considers process operability under different disturbances. The proposed co-design optimization framework is benchmarked on a case study from Flores-Tlacuahuac, et al [12]. The mathematical formulation of this case study for the proposed co-design optimization framework is explained in Section 3. Section 4. discusses the implementation techniques. Section 5. presents the results of the proposed optimization framework while Section 6. provides assessment, discussion and explanation of the results and comparison with the conventional framework .

\section{Methodology}

This section presents the mathematical programming and solving methodology for the co-design of chemical processes. Section 2.1 reviews the conventional optimization framework for co-design. which uses the combined model of the process and its controllers. Its mathematical statement is presented in Section 2.2. The application of the inversely controlled process model and its relation to the notion of perfect control is discussed in Section 2.3. The inversely controlled process model is then embedded in the conventional optimization framework. The objective function of co-design optimization under uncertainty is discussed in Section 2.4.

\subsection{Conventional optimization framework for integrated design and control of chemical processes}

The co-design framework includes an objective function to screen between alternative designs. The model is a set of differential algebraic equations with equality or inequality constraints and is used by the optimization algorithm to determine both structural and parametric variables. While structural decisions concern choices between different process technologies and different control structures, the parametric decisions concern the values of process variables such as flows, temperatures or pressures and also controller parameters.

The conventional practice is to develop a superstructure model which is the combination of all process and sub-process models and their controllers. This combined model provides optimization decision variables which are structural (integer) or parametric (real). The optimizer prunes the superstructure by specifying the structural variables. Simultaneously, the parametric variables of the remaining structure are optimized. This framework is illustrated in Fig. 1. which shows candidate optimization variables being exported by the optimization algorithm to the superstructure of the combined model, which is shown by the dotted envelope. By fixing the values of the optimization variables, the superstructure is reduced to a model of a candidate solution for the co-design problem. Its performance is tested under different disturbances and reported to the optimization algorithm. The optimization algorithm evaluates the termination criteria and decides on improvement of the optimization variables. 


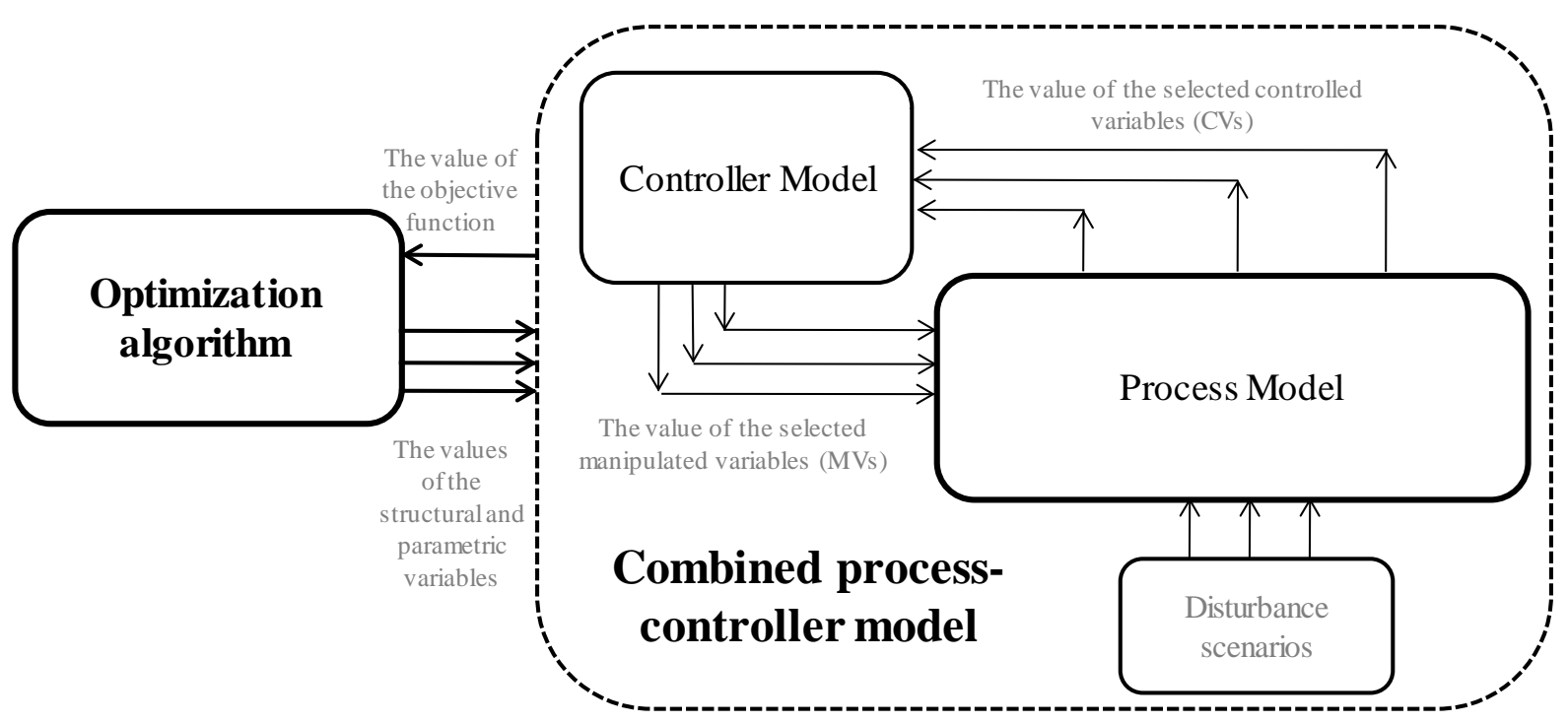

Fig. 1. The conventional optimization framework for integrated design and control of chemical processes

In Fig 1. the optimizer decides on optimization variables for a combined process and controller model. However, modelling of controllers in the optimization framework is undesirable for several reasons:

(i) including decisions on the type of controllers and the tuning parameters increases the problem size; (ii) it is not possible to include some important classes of advance controllers i.e. model predictive controllers (MPCs), in this optimization framework. In practice, these controllers are designed using commercial packages often during process commissioning stages [1, 13]; and (iii) the dynamic optimization frameworks are very sensitive to unstable solutions [10,14] and systematic generation of reasonable initial values for controller parameters is so computationally demanding that it even becomes impossible. Some examples of difficulties with unstable solutions are presented in Sections 6.2.and 6.3.

\subsection{Mathematical problem statement}

The conventional approach to integrated design and control of chemical processes can be formulated as a stochastic mixed integer dynamic optimization problem as follows [4, 13]:

$$
\operatorname{Min} E\left\{\boldsymbol{J}_{\left(\chi_{\boldsymbol{p}}, \chi_{c}, \boldsymbol{p}, \boldsymbol{\vartheta}\right)}\left[\boldsymbol{x}(t), \boldsymbol{z}(t), \boldsymbol{u}(t), \boldsymbol{y}(t), \zeta(t), \boldsymbol{\gamma}(t), \chi_{\boldsymbol{p}}, \chi_{\boldsymbol{c}}, \boldsymbol{p}, \boldsymbol{\vartheta}, \boldsymbol{\mu}(t)\right]\right\} \quad \text { Problem I }
$$

Subject to:

$$
\begin{gathered}
\boldsymbol{f}\left[\dot{\boldsymbol{x}}(t), \boldsymbol{x}(t), \boldsymbol{z}(t), \boldsymbol{u}(t), \boldsymbol{y}(t), \chi_{\boldsymbol{p}}, \boldsymbol{p}, \boldsymbol{z}(t)\right]=0 \\
\boldsymbol{h}\left[\boldsymbol{x}(t), \boldsymbol{z}(t), \boldsymbol{u}(t), \boldsymbol{y}(t), \chi_{\boldsymbol{p}}, \boldsymbol{p}\right]=0 \\
\boldsymbol{g}\left[\boldsymbol{x}(t), \boldsymbol{z}(t), \boldsymbol{u}(t), \boldsymbol{y}(t), \chi_{\boldsymbol{p}}, \boldsymbol{p}\right] \leq 0 \\
\boldsymbol{\theta}\left[\dot{\zeta}(t), \zeta(t), \boldsymbol{\gamma}(t), \boldsymbol{x}(t), \boldsymbol{z}(t), \boldsymbol{y}(t), \boldsymbol{u}(t), \chi_{\boldsymbol{c}}, \boldsymbol{\vartheta}\right]=0 \\
\boldsymbol{\varphi}\left[\boldsymbol{x}(t), \boldsymbol{u}(t), \boldsymbol{y}(t), \zeta(t), \boldsymbol{\gamma}(t), \chi_{\boldsymbol{c}}, \boldsymbol{\vartheta}\right]=0 \\
\Omega[\boldsymbol{\mu}(t)]=0
\end{gathered}
$$

In the above, $\boldsymbol{x}(t)$ is the vector ${ }^{1}$ of process differential variables, $\boldsymbol{z}(t)$ is the vector of process algebraic variables, $\boldsymbol{u}(t)$ is the vector of manipulated variables, $\boldsymbol{y}(t)$ is the vector of candidate controlled variables, $\boldsymbol{p}$ is the vector of process parameters, $\zeta(t)$ is the vector of control differential variables (e.g. PID control law), $\boldsymbol{\gamma}(t)$ is the vector of control algebraic variables , $\boldsymbol{\vartheta}$ is the vector of

\footnotetext{
${ }^{1}$ In this article, bold characters are reserved for vectors, and italic characters are scalar.
} 
control parameters, $\boldsymbol{\mu}(t)$ is the vector of stochastic disturbance parameters, $\chi_{\boldsymbol{p}}$ is the vector of structural process variables, $\chi_{\boldsymbol{c}}$ is the vector of structural control variables. While $\chi_{\boldsymbol{p}}$ and $\chi_{\boldsymbol{c}}$ are vectors of integer variables, the rest of variables are real.

In addition, $\boldsymbol{f}[]=0$ is the vector of process differential equations, $\boldsymbol{h}[]=0$ is the vector of process algebraic equations, $\boldsymbol{g}[] \leq 0$ is the vector inequality constraints, $\boldsymbol{\theta}[]=0$ is the vector of control differential equations, $\varphi[]=0$ is the vector of control algebraic equations, $\Omega[$ ] $=0$ is the vector of equations for disturbances. The expected value $E\{\}$ of the dynamic objective function $J[]$ should be minimized.

The case study in Section 3 gives physical examples of each of these categories of variables and equations.

\subsection{A novel optimization framework using inversely controlled process model}

The aim of the analysis in this sub-section is to disentangle the complexities associated with controllers from the conventional optimization framework. The modification is firstly explained by considering troublesome elements in Fig. 1. Then, Problem I above is modified to eliminate the need for modelling of the controllers. The schematic of the new optimization framework is presented in Fig. 2.

\subsubsection{Perfect control and the inversely controlled process model}

The best possible controller is a perfect controller that would generate manipulated variables taking account of disturbances such that the controlled variables are precisely at their specified setpoints. As shown in [16] the best possible controller can be determined by the inverse solution of the process model. This is a well-known concept [17] that resulted in development of a class of controllers which use the inverse of the process model as an internal element of the controller [18]. However, no attempt has been made to integrate the concept of perfect control into the co-design problem. This paper addresses this opportunity.

There will generally be more than one perfect controller because it is possible to select different controlled variables and manipulated variables within the available degrees of freedom. The alternative control structures can be compared in a systematic way using an optimization framework.

Fig. 2. shows the concept. The model of the controller has been replaced with an equation representing perfect control which enables the directions of the information flows in the optimization framework to be reversed for the controlled variables (CVs) and the manipulated variables (MVs). The inversely controlled model is a superstructure for alternative process and control structures and must be pruned by the optimization algorithm. 


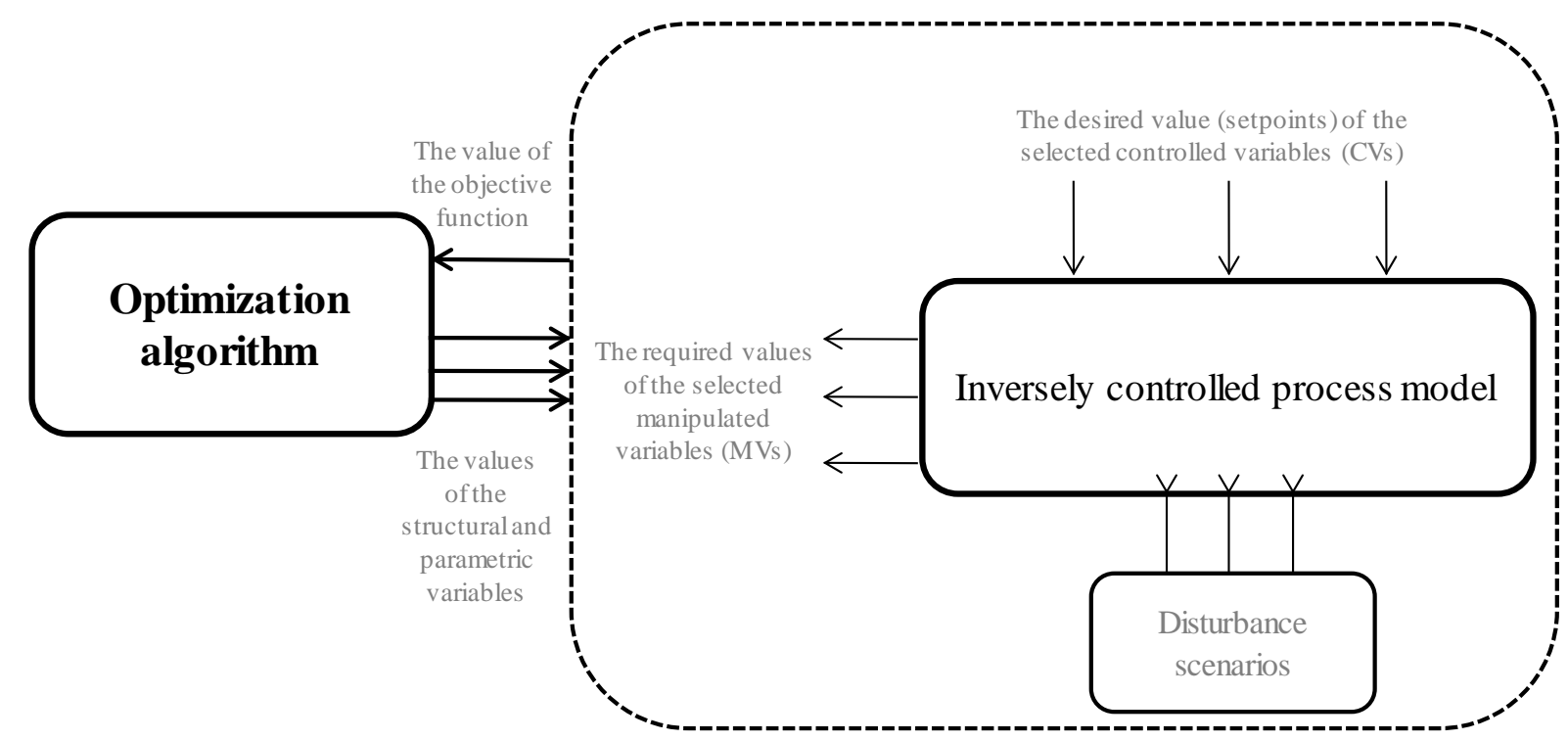

Fig 2. The proposed optimization framework for integrated design and control of chemical processes using the inversely controlled process model

The information flow in Fig. 2. is as follows. Firstly, the optimization algorithm decides on candidate values of the parametric and structural optimization variables. These variables include the sizes of the process equipments and the operating conditions such as temperatures, pressures and flow rates as well as the process structure and the control structure. The values of controlled variables are maintained constant while the time-dependent trajectories of the manipulated variables are adjusted in order to reject the disturbances. Then, the values of the objective function and constraints can be evaluated and reported to the optimization algorithm.

\subsubsection{Mathematical formulation of the inversely controlled process model}

In order to disentangle the design of controllers, their algebraic and differential equations $(\boldsymbol{\theta}[]=0$ and $\varphi[]=0$ ) must be removed from the mathematical formulation of Problem I. They are replaced by new equations which describe perfect control. The values of the manipulated variables for the perfect control are calculated from the controlled variables; hence the desired values of controlled variables should be maintained constant by the new equations:

$$
y_{i}(t)=\eta_{i}
$$

where $\boldsymbol{y}_{\boldsymbol{i}}(t)$ is the selected controlled variable and $\eta_{i}$ is the set point. In principle, $\eta_{i}$ can be time dependent, however in the optimization of a continuous process it would normally be constant. The modified formulation of the optimization framework is as follows:

$$
\operatorname{Min} E\left\{\boldsymbol{J}_{\left(\chi_{\boldsymbol{p}}, \chi_{c}, \boldsymbol{p}, \boldsymbol{\eta}\right)}\left[\boldsymbol{x}(t), \boldsymbol{z}(t), \boldsymbol{u}(t), \boldsymbol{y}(t), \boldsymbol{\mu}(t), \chi_{\boldsymbol{p}}, \chi_{c}, \boldsymbol{p}, \boldsymbol{\eta}_{\boldsymbol{i}}\right]\right\} \quad \text { Problem II }
$$

subject to:

$$
\begin{gathered}
\boldsymbol{f}\left[\dot{\boldsymbol{x}}(t), \boldsymbol{x}(t), \boldsymbol{z}(t), \boldsymbol{u}(t), \boldsymbol{y}(t), \chi_{\boldsymbol{p}}, \boldsymbol{p}\right]=0 \\
\boldsymbol{h}\left[\boldsymbol{x}(t), \boldsymbol{z}(t), \boldsymbol{u}(t), \boldsymbol{y}(t), \chi_{\boldsymbol{p}}, \boldsymbol{p}\right]=0 \\
\boldsymbol{g}\left[\boldsymbol{x}(t), \boldsymbol{z}(t), \boldsymbol{u}(t), \boldsymbol{y}(t), \chi_{\boldsymbol{p}}, \boldsymbol{p}\right] \leq 0 \\
\boldsymbol{y}(t)-\boldsymbol{\eta}=0 \\
\Omega[\boldsymbol{\mu}(t)]=0
\end{gathered}
$$


Since the control structure is being simultaneously decided during co-design optimization, different controlled variables may be selected during the optimization search, which represent different perfect controls. Each candidate control structure generates a different set of DAEs.

\subsubsection{Practical considerations}

The result of the new co-design framework is optimal according to the objective function in Problem II above. Due to satisfaction of Eqn. (1), the integral of the square of controller error (ISE) will be equal to zero, and the control is perfect:

$$
I S E_{i}=\int\left[\boldsymbol{y}_{\boldsymbol{i}}(t)-\boldsymbol{\eta}_{\boldsymbol{i}}\right]^{2} d t=0
$$

Hence, the design gives the best possible controller coupled with the optimal control structure. Questions arise, however, about (i) whether the perfect control exists, and (ii) what practical use can be made of the results.

Optimization with perfect control may fail if inversion of the process model is not possible. Russell [19] summarized the scenarios in which the inversion of a process model is limited. These are right half plane zeros or unstable zero dynamics for nonlinear systems [16], model uncertainties, manipulated variables constraints and time delays. Fortunately, none of these concerns limits the application of the inverse process model concept. The reason is that the process model is represented as inequality and equality constraints in the optimization framework. Hence, if the inversion of the process model is not possible for a candidate solution, the constraints are violated which directs the optimization algorithm towards other candidates that do make the process functionally controllable. Furthermore, the simultaneous solving strategy for mixed integer dynamic optimization is not limited by time delays as all variables are fully parameterized and being solved simultaneously, [14, 20].

Perfect control is rarely achievable in practice, and the practical design and implementation of the controller is deferred to the control engineering practitioners. While the structure and target closed loop performance are specified by the optimization framework, it is for the control engineer to devise a practical controller which most closely meets the performance from the range of controller types available to him or her. Such a design philosophy is consistent with industrial practices developed over the last 20 years $[21,22]$ in which the fitness for purpose of a control loop is assessed against the best achievable performance.

\subsection{Objective function for co-design optimization under uncertainty}

As mentioned, the perfect control is not unique. Control structures comprising different selections of manipulated and controlled variables may result in different perfect controls. Screening and selecting the optimal control structures require a criterion, which is the objective function in the first line of Problem II in the optimization framework. Luyben [2] described competing objective functions for integrated design and control. He stated that the objective function of the co-design problem must capture both steady-state economy and dynamic performance of the process.

The value of the objective function depends on the disturbances [11]. Therefore, co-design should be formulated as a stochastic optimization problem. The variables of such a problem consist of the firststage variables, the uncertain variables and the second-stage (also called recourse) variables. The firststage variables must be decided before the uncertain variables are realized. Once the uncertain variables present themselves, the second-stage variables are adjusted to minimize the objective function [23].

In the context of this article, the uncertain variables (e.g. disturbances in the feed composition in the case study of Section 3) divide the variables into first-stage and second-stage variables. Example of first-stage variables in the co-design problem are the process structure, process parameters and the control structure (i.e., selection of controlled and manipulated variables). Examples of second-stage variables are the values of the manipulated variables which must be adjusted according to disturbances. 


\section{Case study for the conventional co-design optimization framework}

Flores-Tlacuahuac, et al [12] studied a process comprising of two series reactors in order to benchmark the performance of different solving methodologies for mixed integer dynamic optimization. The cooling system of the process may have either co-current or counter-current heat exchangers which are shown in Figs. 3a,b. respectively. The mathematical formulation of their study is presented in this section and matches the optimization framework of Fig 1. In Section 4., this mathematical formulation will be modified and adapted to the new optimization framework using an inversely controlled process model.

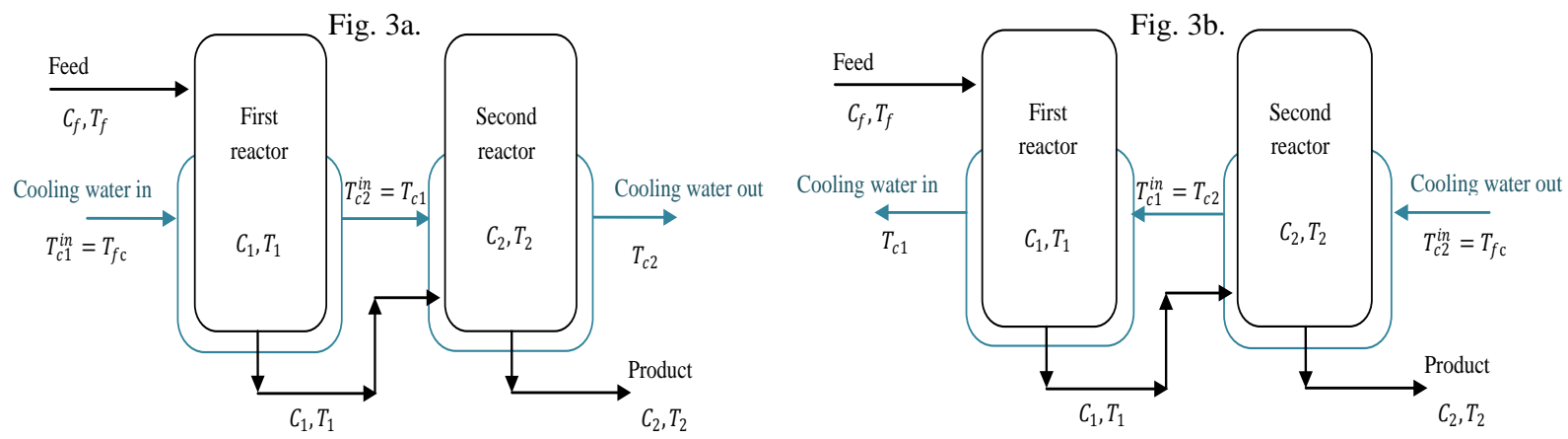

Figs. 3. Different process structures: a) the co-current structure b) the counter-current structure.

The mass and energy balances of the first and the second reactors are presented by Eqns. (3) to (8). The definition of the variables and the value of the design parameters are reported in Table 1, [12]. The mass and energy balances for the first reactor are:

$$
\begin{aligned}
& \frac{d C_{1}}{d t}=\frac{C_{f}-C_{1}}{\theta_{1}}+r_{A 1} \\
& \frac{d T_{1}}{d t}=\frac{T_{f}-T_{1}}{\theta_{1}}+\beta \times r_{A 1}-\alpha_{1} \times\left(T_{1}-T_{c 1}\right)
\end{aligned}
$$

The energy balance for the cooling jacket of the first reactor is:

$$
\frac{d T_{c 1}}{d t}=\frac{T_{C 1}^{i n}-T_{C 1}}{\theta_{c 1}}+\alpha_{c 1} \times\left(T_{1}-T_{c 1}\right)
$$

The mass and energy balances for the second reactor are:

$$
\begin{aligned}
& \frac{d C_{2}}{d t}=\frac{C_{1}-C_{2}}{\theta_{2}}+r_{A 2} \\
& \frac{d T_{2}}{d t}=\frac{T_{1}-T_{2}}{\theta_{2}}+\beta \times r_{A 2}-\alpha_{2} \times\left(T_{2}-T_{c 2}\right)
\end{aligned}
$$

The energy balance for the cooling jacket of the second reactor is:

$$
\frac{d T_{c 2}}{d t}=\frac{T_{c 2}^{i n}-T_{c 2}}{\theta_{c 2}}+\alpha_{c 2} \times\left(T_{2}-T_{c 2}\right)
$$

The parameters in Eqns. (3)-(8) are:

$$
\begin{gathered}
\theta_{1}=\frac{V_{1}}{Q}, \quad \theta_{2}=\frac{V_{2}}{Q}, \quad \alpha_{1}=\frac{U \times A_{1}}{\rho \times V_{1} \times C_{p}}, \quad \alpha_{2}=\frac{U \times A_{2}}{\rho \times V_{2} \times C_{p}} \\
\theta_{c 1}=\frac{V_{c 1}}{Q_{c}}, \quad \theta_{c 2}=\frac{V_{c 2}}{Q_{c}}, \quad \alpha_{c 1}=\frac{U \times A_{1}}{\rho_{c} \times V_{c 1} \times C_{p c}}, \quad \alpha_{c 2}=\frac{U \times A_{2}}{\rho_{c} \times V_{c 2} \times C_{p c}}
\end{gathered}
$$




$$
r_{A 1}=-K_{0} \times e^{-E / R \times T_{1}} \times C_{1}, \quad r_{A 2}=-K_{0} \times e^{-E / R \times T_{2}} \times C_{2}, \quad \beta=\frac{\Delta H r}{\rho \times C_{p}},
$$

The structural process decision regarding the process structure is represented by the integer variable $\chi_{p}$ in the Eqns. (9) and (10):

$$
\begin{aligned}
& T_{c 1}^{i n}= \chi_{p} \times T_{f \mathrm{c}}+\left(1-\chi_{p}\right) \times T_{c 2} \\
& T_{c 2}^{i n}=\chi_{p} \times T_{c 1}+\left(1-\chi_{p}\right) \times T_{f \mathrm{c}} \\
&\left\{\begin{array}{lr}
\chi_{p}=1 & \text { co-current configuration } \\
\chi_{p}=0 & \text { countercurrent configuration }
\end{array}\right.
\end{aligned}
$$

These equations ensure that in the co-current structure, $T_{c 1}^{i n}$ is equal to $T_{f \mathrm{c}}$ and $T_{c 2}^{i n}$ is equal to $T_{c 1}$ and in the counter-current structure, $T_{c 1}^{i n}$ is equal to $T_{c 2}$ and $T_{c 2}^{i n}$ is equal to $T_{f \mathrm{c}}$.

The equations for the controller model are:

$$
\begin{aligned}
& T_{f}=T_{f, \text { nominial }}+\left(1-\chi_{m v}\right) \times\left(K_{P} \times P(t)+K_{i} \times I(t)\right) \\
& Q_{c}=Q_{c, \text { nominial }}-\chi_{m v} \times\left(K_{P} \times P(t)+K_{i} \times I(t)\right) \\
& P(t)=\chi_{c v} \times\left(T_{1, \text { setpoint }}-T_{1}\right)+\left(1-\chi_{c v}\right) \times\left(T_{2, \text { setpoint }}-T_{2}\right) \\
& \frac{d I(t)}{d t}=P(t), \quad I(0)=0
\end{aligned}
$$

The tuning parameters of the controller, $K_{P}$ and $K_{i}$, are optimization variables in the conventional framework. Integer variable $\chi_{m v}$ selects between candidate manipulated variables, which are the flowrate of the cooling water $Q_{c}$, or the temperature of the feed $T_{f}$. Integer variable $\chi_{c v}$ selects between the candidate controlled variable, which are the temperature of the first reactor $T_{1}$, or the temperature of the second reactor $T_{2}$.

In the original case study presented by Flores-Tlacuahuac, et al [12], the following objective function was introduced:

$$
\operatorname{Min} \frac{1}{t_{\text {final }}} \int_{0}^{t \text { final }}\left(T_{2, \text { setpiont }}-T_{2}\right)^{2} d t
$$


Table 1

The parameters and the values of the variables at the base case scenario

\begin{tabular}{|c|c|c|c|c|c|}
\hline Parameter & Description & Value [12] & Unit [12] & Value & SI Unit \\
\hline$Q$ & Volumetric feed flow rate & 2.5 & L.s s $^{-1}$ & $2.5 \times 10^{-3}$ & $\mathrm{~m}^{3} \cdot \mathrm{s}^{-1}$ \\
\hline$T_{f}$ & Feed stream temperature & 29 & ${ }^{\circ} \mathrm{C}$ & 302 & K \\
\hline$C_{f}$ & Feed Stream concentration & 0.6 & mol.L $L^{-1}$ & 0.6 & kmol.m ${ }^{-3}$ \\
\hline$V_{1}$ & Volume of the first reactor & 900 & L & 0.9 & $\mathrm{~m}^{3}$ \\
\hline$V_{2}$ & $\begin{array}{c}\text { Volume of the second } \\
\text { reactor }\end{array}$ & 900 & $\mathrm{~L}$ & 0.9 & $\mathrm{~m}^{3}$ \\
\hline$Q_{c}$ & Cooling water flow rate & 2 & L.s. $s^{-1}$ & $2 \times 10^{-3}$ & $\mathrm{~m}^{3} \cdot \mathrm{s}^{-1}$ \\
\hline$T_{f \mathrm{c}}$ & $\begin{array}{l}\text { Cooling water feed stream } \\
\text { temperature }\end{array}$ & 25 & ${ }^{\circ} \mathrm{C}$ & 298 & $\mathrm{~K}$ \\
\hline$V_{c 1}$ & $\begin{array}{l}\text { Volume of the cooling } \\
\text { jacket of the first reactor }\end{array}$ & 100 & $\mathrm{~L}$ & 0.1 & $\mathrm{~m}^{3}$ \\
\hline$V_{c 2}$ & $\begin{array}{l}\text { Volume of the cooling } \\
\text { jacket of the second reactor }\end{array}$ & 100 & $\mathrm{~L}$ & 0.1 & $\mathrm{~m}^{3}$ \\
\hline$E$ & Activation energy & 10.1 & kcal.mol ${ }^{-1}$ & $4.2 \times 10^{7}$ & $\mathrm{~J} \mathrm{kmol}^{-1}$ \\
\hline$K_{0}$ & Pre-exponential factor & 2000 & $\mathrm{~s}^{-1}$ & 2000 & $\mathrm{~s}^{-1}$ \\
\hline$R$ & Ideal gas constant & 0.00198 & kcal.mol ${ }^{-1} \cdot \mathrm{K}^{-1}$ & $8.32 \times 10^{3}$ & $\mathrm{~J} . \mathrm{kmol}^{-1} \cdot \mathrm{K}^{-1}$ \\
\hline$\rho$ & Products density & 850 & g. $\mathrm{L}^{-1}$ & 850 & $\mathrm{~kg} \cdot \mathrm{m}^{-3}$ \\
\hline$C_{p}$ & Product heat capacity & 0.000135 & $\mathrm{kcal} \cdot \mathrm{g}^{-1} \cdot \mathrm{C}^{-1}$ & 564.84 & $\mathrm{~J} . \mathrm{kg}^{-1} \cdot \mathrm{C}^{-1}$ \\
\hline$\Delta H_{r}$ & Heat of reaction & -35 & kcal.mol ${ }^{-1}$ & $-1.46 \times 10^{8}$ & J.kmol ${ }^{-1}$ \\
\hline$\rho_{c}$ & Cooling water density & 1000 & g..$^{-1}$ & 1000 & kg. $\mathrm{m}^{-3}$ \\
\hline$C_{p c}$ & Cooling water heat capacity & 0.001 & $\mathrm{kcal} \cdot \mathrm{g}^{-1} \cdot \mathrm{C}^{-1}$ & $4.2 \times 10^{3}$ & $\mathrm{~J} . \mathrm{kg}^{-1} \cdot \mathrm{K}^{-1}$ \\
\hline$A$ & Heat transfer area & 900 & $\mathrm{~cm}^{2}$ & 0.09 & $\mathrm{~m}^{2}$ \\
\hline$U$ & Heat transfer coefficient & 0.00004 & kcal.s ${ }^{-1} \cdot \mathrm{cm}^{-2} \cdot \mathrm{C}^{-1}$ & $1.7 \times 10^{3}$ & $\mathrm{~J} \cdot \mathrm{s}^{-1} \cdot \mathrm{m}^{-2} \cdot \mathrm{K}^{-1}$ \\
\hline
\end{tabular}

\section{Application of co-design optimization using an inversely controlled process model}

This section applies the proposed co-design optimization framework to the case study. In Section 4.1., the case study is extended, and the selection of disturbances is justified. Section 4.2. presents the formulation of the inversely controlled process model. The objective function of the co-design framework is discussed in Section 4.3 and a solving strategy is suggested in Section 4.4. Section 4.5 explores the issues of controllability of candidate solution, and Section 4.6 describes a method for comparison of a combined process-controller model with the inversely controlled process model for the same process.

\subsection{Amendments to the original case study}

[12] considered a fixed value for the heat transfer area $A_{i}, i=1,2$ of each cooling jacket. However, it is usual to scale the heat transfer area of a cooling jacket with reactor volume by:

$$
A_{i}=\operatorname{coef} \times\left(V_{i}\right)^{2 / 3}
$$

Therefore Eqn. (16) is added to the original case study and its coefficient is calculated from the basecase design, shown in Table. 1 , resulting in coef $=9.655 \mathrm{~cm}^{2} \cdot \mathrm{L}^{(-2 / 3)}$. The base case design requires a heat transfer area that is much smaller than the surface area of the reactor. Such a configuration would have to be realized in practice by a jacket that makes only partial contact with the reactor walls. 
Flores-Tlacuahuac, et al [12] suggested 50\% and 200\% as the lower and upper bounds for the optimization values. The upper and lower bounds for the optimization variables used instead in this paper are $50 \%$ and $300 \%$. The reason is that for some specific structures the heat transfer is thermodynamically limited by the maximum allowable temperature of the cooling water exiting the process, which is $80^{\circ} \mathrm{C}$.

Flores-Tlacuahuac, et al [12] assumed the disturbance to be in the feed composition. They evaluated several disturbances in the range $C_{f}=0.55 \mathrm{kmol} . \mathrm{m}^{-3}$ to $C_{f}=0.65 \mathrm{kmol} . \mathrm{m}^{-3}$ with different time constants.

The present research considers optimization under uncertainty based on the likelihood of disturbances. Once a process is operating, the likelihood of a disturbance can be extracted from previous operational data, or from a pilot plant. It can also include the most likely operational conditions based on the distribution of important economic variables, different feedstock specifications and products grades. In this research a step disturbance from $C_{f}=0.55 \mathrm{kmol} . \mathrm{m}^{-3}$ to $C_{f}=0.65 \mathrm{kmol} . \mathrm{m}^{-3}$ is considered a likely operational condition. This disturbance covers all the operational regions explored by the disturbances in the original case study [12]. However, due to nonlinearity of the process the direction of the disturbance may be important. Therefore, this paper also considers another disturbance with the same magnitude but the reverse direction from $C_{f}=0.65 \mathrm{kmol}^{-3}$ to $C_{f}=0.55$ $\mathrm{kmol} . \mathrm{m}^{-3}$. It is assumed that these disturbances have the same likelihood. Thus the overall objective function is the average of the objective functions for both disturbances.

\subsection{Inversely controlled process model for the case of two series reactors}

This section discusses replacement of the controller model with the perfect control Eqn. (1) and inverting the process model. The inversely controlled process model for the case of series reactors consist of Eqns. (3-10) and the following equations.

The structural control decision regarding the selection of controlled variables is represented by integer variable $\chi_{c v}$ as follows:

$$
\begin{array}{r}
\chi_{c v} \times T_{1}+\left(1-\chi_{c v}\right) \times T_{2}=\chi_{c v} \times T_{1, \text { setpoint }}+\left(1-\chi_{c v}\right) \times T_{2, \text { setpoint }} \\
\begin{cases}\chi_{c v}=1 & T_{1} \text { is selected as controlled variable } \\
\chi_{c v}=0 & T_{2} \text { is selected as controlled variable }\end{cases}
\end{array}
$$

The structural control decision regarding the selection of manipulated variables is represented by integer variable $\chi_{m v}$ as follows:

$$
\begin{gathered}
\chi_{m v} \times T_{f}+\left(1-\chi_{m v}\right) \times Q_{c}=\chi_{m v} \times T_{f, \text { nominial }}+\left(1-\chi_{m v}\right) \times Q_{c, \text { nominial }} \\
\begin{cases}\chi_{m v}=1 & Q_{c} \text { is selected as manipulated variable } \\
\chi_{m v}=0 & T_{f} \text { is selected as manipulated variable }\end{cases}
\end{gathered}
$$

These equations ensure that when a manipulated variable is selected, the other candidate manipulated variable is maintained constant at its nominal value and the value of the selected manipulated variable is free and available to the DAE solver.

\subsection{Objective function}

In the original case study in [12], the objective function of the co-design optimization was equation (15). This objective function is controller oriented, in that the process design should be changed by the optimizer in order to minimize a measure of the controller error (e.g. ISE).

Luyben [2] presented a discussion of competing objective functions of co-design. He described a conflict between the economic and controllability of chemical processes. While the economic objectives such as investment capital or energy costs favour thermodynamic reversibility, the 
controllability objectives prefer to increase thermodynamic irreversibility. He gave examples where improving the steady state economic objectives limits the dynamic control performance, for instance a small heat transfer area in an isothermal exothermic reactor that may limit controllability of the process. In this research, the following objective function is considered for the case study of two tanks in series:

$$
\begin{gathered}
\operatorname{Min} \sum_{k=1,2} L_{k} \times J\left(\mu_{k}\right) \\
J\left(\mu_{k}\right)=\left\{\alpha \times \int_{t 0}^{t f i n a l}\left|C_{2}-C_{2, \text { target }}\right| d t+\beta \times \int_{t 0}^{t f \text { inal }}\left|M V-M V_{\text {nominal }}\right| d t+\sigma \times\right. \\
\left.\int_{t 0}^{t f \text { inal }} \frac{|d M V|}{d t} d t+\sum_{i=1}^{2}(P \times \text { Vreactor, } i+P c \times \text { Vjackect, } i)\right\}
\end{gathered}
$$

The combined scalar objective value (Eqn. 19) is constructed using the likelihood of the disturbance (i.e. uncertain variables). In addition, the proposed objective function establishes a trade-off between steady-state economy and dynamic performance of chemical processes.

The likelihoods of disturbances are equal, i.e., $L_{\mu=1,2}=0.5$. The value of $t_{f i n a l}=1000 \mathrm{~s}$ is large enough that if a candidate design is feasible, the process would reach a new steady state. The target value of the composition of the second reactor $C_{2, \text { target }}$ was set to zero in the first integral to maximize the conversion. The implication of the second integral is that when disturbances are imposed to the optimal design, maintaining the controlled variable at its setpoint requires minimum changes in the value of manipulated variable [13,24]. The third term concerns the rate of change in the manipulated variable, and its coefficient influences how fast a manipulated variable can perform a control action [13]. The fourth term is the summation of the required capital investment for purchasing reactors and their cooling jackets which are proportional to their volumes. In the proposed objective function, the first, second and third terms represent the dynamic controllability of the process and the fourth term represents the steady-state economics.

No economic data are available in [12] for the case study. Hence, in this article some weighting factors are introduced in order to establish a trade-off between competing objectives. These factors represent orders of magnitude of costs. The pricing factors of reactors and cooling jackets are set to $P=0.001, P c=0.0015$, respectively. The cooling jackets are more expensive because they are more prone to thermal shocks, erosion and corrosion and have higher manufacturing costs due to their shape, size and hydraulic considerations (e.g. low pressure drop, high heat transfer area). Values are assumed for $\alpha=1$ and $\beta=0.0001$, and $\sigma=100$. Both $\beta$ and $\sigma$ are scaled by the nominal value of the manipulated variables.

\subsection{Solving strategies and implementation techniques}

This section presents the solving method for Problem II. It is an initial value problem of a type known as a Bolza problem, i.e., the objective function is a function of both final time and the integral over the simulation time, [14]. In this research, the sequential dynamic optimization strategy is used for solving the problem, as follows. The equivalent discretized version of Problem II is:

$$
\text { Min } \sum_{k=1}^{N_{\mu}} L_{k} \times J_{k}\left(\boldsymbol{\mu}_{k}\right) \quad \text { Problem III-a }
$$

subject to:

$$
\begin{gathered}
\boldsymbol{J}\left(\boldsymbol{\mu}_{k}\right)=\sum_{l=1}^{N_{T}} \boldsymbol{J}^{l}\left[\boldsymbol{x}\left(t^{l}\right), \boldsymbol{z}\left(t^{l}\right), \boldsymbol{u}\left(t^{l}\right), \boldsymbol{y}\left(t^{l}\right), \mu_{k}\left(t^{l}\right), \boldsymbol{\eta}_{\boldsymbol{i}}, \chi_{\boldsymbol{p}}, \chi_{\boldsymbol{c}}, \boldsymbol{p}\right] \\
\boldsymbol{f}\left[\dot{\boldsymbol{x}}\left(t^{l}\right), \boldsymbol{x}\left(t^{l}\right), \boldsymbol{z}\left(t^{l}\right), \boldsymbol{u}\left(t^{l}\right), \boldsymbol{y}\left(t^{l}\right), \chi_{\boldsymbol{p}}, \boldsymbol{p}\right]=0 \\
\boldsymbol{h}\left[\boldsymbol{x}\left(t^{l}\right), \boldsymbol{z}\left(t^{l}\right), \boldsymbol{u}\left(t^{l}\right), \boldsymbol{y}\left(t^{l}\right), \chi_{\boldsymbol{p}}, \boldsymbol{p}\right]=0
\end{gathered}
$$




$$
\begin{gathered}
\boldsymbol{g}\left[\boldsymbol{x}\left(t^{l}\right), \boldsymbol{z}\left(t^{l}\right), \boldsymbol{u}\left(t^{l}\right), \boldsymbol{y}\left(t^{l}\right), \chi_{\boldsymbol{p}}, \boldsymbol{p}\right] \leq 0 \\
\boldsymbol{y}_{\boldsymbol{i}}\left(t^{l}\right)-\boldsymbol{\eta}_{\boldsymbol{i}}=0 \\
\Omega\left[\boldsymbol{\mu}\left(t^{l}\right)\right]=0
\end{gathered}
$$

where $J$ is the value of the objective function (e.g. Eqn. 19) for each uncertain (disturbance) scenario, $\mu . N_{\mu}$ is the number of uncertain scenarios and $L_{\mu}$ is the likelihood of each uncertain (disturbance) scenario. $N_{T}$ is the number of periods (time intervals). In a combined process and controller model (Fig. 1.), input profiles, $\boldsymbol{u}(t)$, are parameterized with coefficients that determine the optimal profile. However, in the inversely controlled process model (Fig. 2.), the controlled variables $\boldsymbol{y}(t)$ (outputs of the forward problem) represent the input variables of the mixed integer dynamic optimization problem and are parameterized by the desired setpoints, i.e., $\boldsymbol{\eta}$ using Eqn. (1). The optimization decisions in problem III-a are all time independent, and this problem can be represented as:

$$
\operatorname{Min} O\left(\boldsymbol{\eta}_{i}, \chi_{p}, \chi_{c}, \boldsymbol{p}\right) \quad \text { Problem III-b }
$$

subject to:

$$
\begin{array}{r}
\boldsymbol{H}\left[\boldsymbol{\eta}_{\boldsymbol{i}}, \chi_{\boldsymbol{p}}, \chi_{\boldsymbol{c}}, \boldsymbol{p}\right]=0 \\
\boldsymbol{G}\left[\boldsymbol{\eta}_{i}, \chi_{\boldsymbol{p}}, \chi_{c}, \boldsymbol{p}\right] \leq 0
\end{array}
$$

where $\boldsymbol{G}$ and $\boldsymbol{H}$ capture all the inequality and equality constraints. The objective function and constraints in Problems III a-b are implicit functions of time-independent variables, and the DAE system is solved in an inner loop using sequential dynamic optimization, [14]. The schematic of the solving methodology is presented in Fig. 4 and explained as follows.

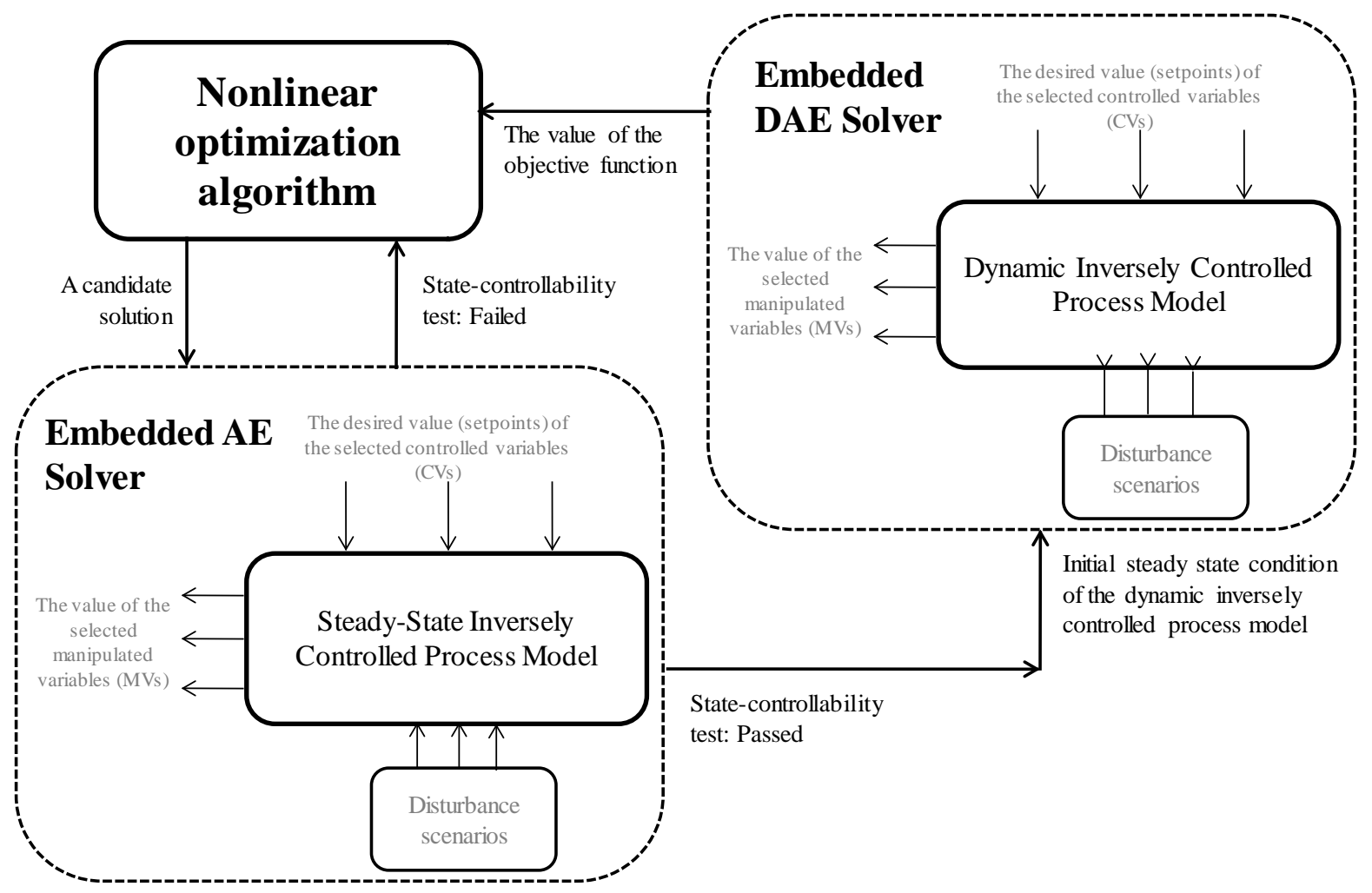

Fig 4. The final proposed co-design optimization framework, including both steady-state and dynamic inversely controlled process models. 
At a given iteration of the optimization cycle, the nonlinear optimization algorithm specifies the candidate values of the time -independent parametric variables. For fixed values of these variables, the Problems III a-b are initial value problems and the DAE system can be solved by numerical integration. The controlled variables are parameterized by Eqn. (1). and the sequential integration gives the time trajectory for the manipulated variables and the remaining state variables. Based on the values of the objective function and the constraints, the optimization algorithm makes decisions regarding the termination of the optimization cycle or improving the values of the optimization variables. The optimization algorithm is not distinguishable and consists of nonlinear optimization algorithm, AE solver, and DAE solvers.

The combinations of the structural decisions regarding the process structure and selection of the manipulated variable and the controlled variable result in eight alternative structures. There are five parametric optimization variables corresponding to the volumes of the reactors and their cooling jackets, and the setpoint for the selected controlled variables. For each of eight alternative structures a nonlinear gradient-based optimization is performed and the optimal values of the parametric optimization variables and the corresponding values of the objective function in Eqn. (19) are reported in Section 6.1.

The integration of DAE system must start from a feasible steady-state condition [25] which is calculated using an inversely controlled steady-state process model (the lower left-hand block in Fig. 4.). It consists of a set of algebraic steady-state equations in which controlled variables are fixed and the values of the rest of variables are calculated using the process model. In the case that a feasible steady-state condition cannot be found, the AE solver reports a failure to the nonlinear optimization algorithm to change the values of optimization variables. This solving strategy saves computational time and efforts by detecting infeasible solutions. This is explained in more detail in the next section.

In this research, the embedded algebraic equation (AE) solver and the embedded differential algebraic equation (DAE) solver were both implemented in Aspen Custom Modeller (ACM), which was invoked in steady-state and dynamic modes, respectively. The optimization algorithm was a nonlinear gradient-based solver code in the Visual Basic Application (VBA) environment. The two software tools were linked using Microsoft COM interface. The co-design optimization framework of Fig. 4 is simulation-optimization with an implicit model [26].

\subsection{Controllability constraints}

This section presents a testing methodology for state and functional controllability. It evaluates steady-state and dynamic controllability constraints.

The concept of testing steady-state controllability is illustrated in Fig. 5, schematically. The graph shows variation of the controlled variable with changes in the manipulated variable at different steady states. The lower profile represents the steady states before a disturbance and the upper profile represents the steady states after the disturbance. The length of a horizontal tie line (dashed line) represents the change in the manipulated variable required to maintain the controlled variable at its set point. A setpoint for a controlled variable is feasible if a horizontal tie-line exists. Conversely, if no horizontal tie-line can be found then the process is not state-wise controllable.

The feasibility of initial and ultimate steady states does not ensure that the transient states are also feasible. An additional test is needed; Fig. 6 . shows the time trajectory of the cooling flowrate due to two disturbances $C_{f}=0.55 \mathrm{kmol} . \mathrm{m}^{-3}$ to $C_{f}=0.65 \mathrm{kmol} . \mathrm{m}^{-3}$ and $C_{f}=0.65 \mathrm{kmol} . \mathrm{m}^{-3}$ to $C_{f}=0.55$ kmol. $\mathrm{m}^{-3}$ ). In this example the volume of reactors are $V_{i}=2.7 \mathrm{~m}^{3}$, and all other process variables are at their nominal points (Table 1.). This figure reveals that although the initial states and final states are feasible, the intermediate states are infeasible. As shown by the shaded area in Fig. 6 . The physical reason is saturation of the control valve and the loss of control action. In this research, path constraints take care of such infeasibilities. They report any constraint violations to the nonlinear optimization algorithm. In addition, Fig 6 . shows that due to the nonlinearity of the process two disturbances with 
opposite directions do not result in symmetrical trajectories. This is the reason that the direction of disturbances must be included in the functional controllability analysis.
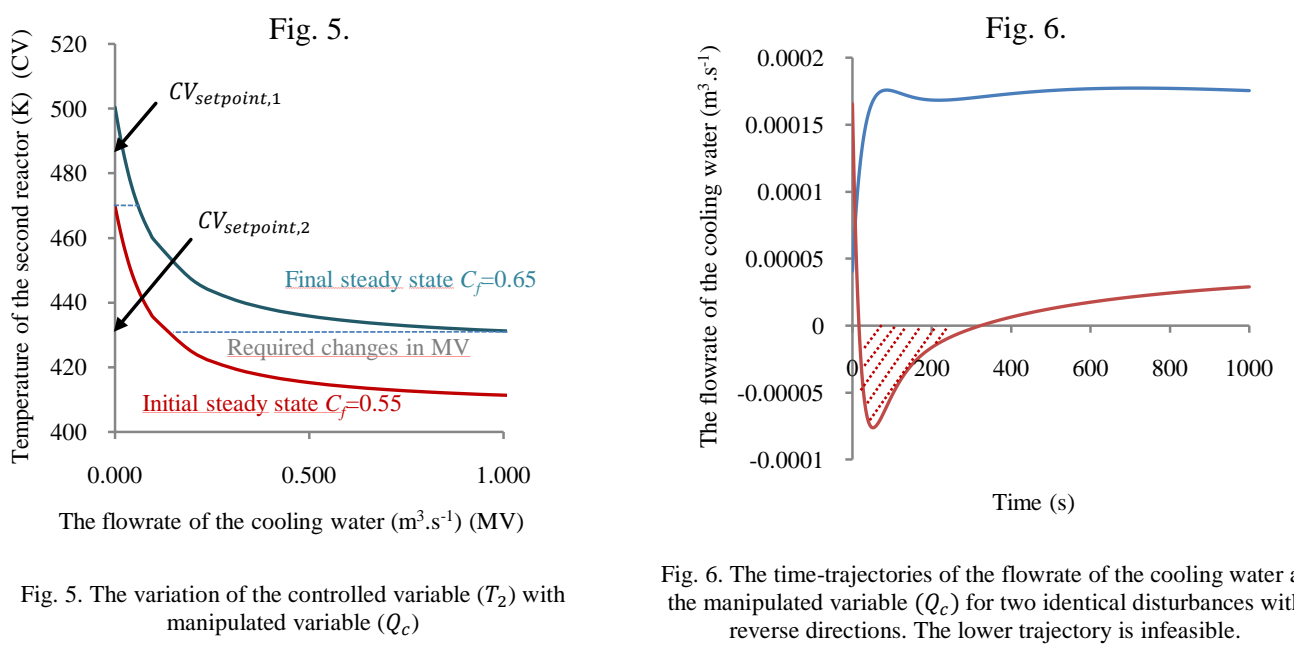

Fig. 6. The time-trajectories of the flowrate of the cooling water as the manipulated variable $\left(Q_{c}\right)$ for two identical disturbances with reverse directions. The lower trajectory is infeasible.

\subsection{Combined process-controller model versus inversely controlled process model}

The authors of [12] reported difficulties in finding a stabilizing PI controller. Therefore a comparison will be made between that problematical case and the same case handled by means of the inverse process model. As reported in [12], the optimized PI controller was unable to bring the process to a steady state and the aim of the comparison is to see if the inversely process model can do better.

The process structure reported in the first part of the results in [12] was counter-current, in which the temperature of the feed was paired with the temperature of the second reactor, and the disturbances was from $C_{f}=0.65 \mathrm{kmol} . \mathrm{m}^{-3}$ to $C_{f}=0.55 \mathrm{kmol} . \mathrm{m}^{-3}$. In that case, the design parameters of the process were not involved in optimization, and only controller parameters were optimized. This structure and disturbance were simulated using the DAE solver in Fig. 4, (i) with the best controller from reference [12] and (ii) using dynamic inversion of the same model with the controlled variables held at their set points according to Eqn. (1). The DAE solver starts from a common steady state and simulates the effect of disturbance on both models. The trajectories of (i) are reconstructed using the reported controller parameters $K_{p}=500$ and $K_{I}=0.357 \mathrm{~s}$ from Table 5 of [12]. The second trajectory is constructed using the inversely controlled process model. The comparisons are presented graphically in the result Section 5. and explained in the Discussion Section 6.2

\section{The results}

This section presents the results of the methods described in Section 4.

There are three structural optimization variables corresponding to the process structure $\chi_{\boldsymbol{p}}$, selection of the manipulated variable $\chi_{m v}$, and selection of the controlled variable $\chi_{c v}$. The combinations of these structural decisions result in eight alternative structures. There are five parametric optimization variables corresponding to the volumes of the reactors and their cooling jackets, and the setpoint for the selected controlled variable. Table 2 reports the optimal values of the parametric optimization variables and the corresponding values of the objective function in Eqn. (21) for each of the eight structures.

Figs. 7-10. show the other results graphically. They are:

- Figs. 7a-c are the time trajectories of the optimal design using the solving strategy of Section 4.4 . 
- Figs. 8a-c. compare the results of the conventional optimization method (from [12]) using a combined process-controller model with the proposed method, called inversely controlled process model. These are reconstructed using embedded ADE solver described in described in Section 4.6. In each figure, two trajectories are illustrated.

- Figs. 9. shows the effect of the feed temperature on the product composition using controllability analysis method described in Section 4.5.

- $\quad$ Figs. 10a-b. explain the uncontrollable structures in Table 2. These are structures where the flowrate of the cooling water is selected as the manipulated variable and the temperature of the feed is selected as the controlled variable. The employed method is described in Section 4.5. and is based on evaluation of state controllability of different structures.

Table 2.

The results of optimization for different structural outcomes

\begin{tabular}{cccccccccc}
\hline & Structure & Structure & Structure & Structure & Structure & Structure & Structure & Structure \\
& $\begin{array}{c}\text { Counter- } \\
\text { current }\end{array}$ & $\begin{array}{c}\text { Counter- } \\
\text { current }\end{array}$ & $\begin{array}{c}\text { Counter- } \\
\text { current }\end{array}$ & $\begin{array}{c}\text { Counter- } \\
\text { current }\end{array}$ & $\begin{array}{c}\text { Co- } \\
\text { current }\end{array}$ & $\begin{array}{c}\text { Co- } \\
\text { current }\end{array}$ & $\begin{array}{c}\text { Co- } \\
\text { current }\end{array}$ & $\begin{array}{c}\text { Co- } \\
\text { current }\end{array}$ \\
& $T_{2}-T_{f}$ & $T_{1}-T_{f}$ & $T_{2}-Q_{c}$ & $T_{1}-Q_{c}$ & $T_{2}-T_{f}$ & $\boldsymbol{T}_{\mathbf{1}}-\boldsymbol{T}_{\boldsymbol{f}}$ & $T_{2}-Q_{c}$ & $T_{1}-Q_{c}$ \\
\hline $\begin{array}{c}\text { Objective } \\
\text { value }\end{array}$ & 4.43 & 4.31 & 27.22 & Infeasible & 4.36 & $\mathbf{4 . 1 8}$ & 66.54 & Infeasible \\
$\begin{array}{c}\text { Constraints } \\
\text { violation }\end{array}$ & No & No & Yes $^{(2)}$ & Yes $^{(1)}$ & No & No & Yes $^{(2)}$ & Yes $^{(1)}$ \\
$\chi_{p}$ & 0 & 0 & 0 & 0 & 1 & $\mathbf{1}$ & 1 & 1 \\
$\chi_{c v}$ & 0 & 1 & 0 & 1 & 0 & $\mathbf{1}$ & 0 & 1 \\
$\chi_{\text {mv }}$ & 0 & 0 & 1 & 1 & 0 & $\mathbf{0}$ & 1 & 1 \\
$V_{1}$ & 1014 & 1174 & 2501 & - & 1025 & $\mathbf{9 0 2}$ & 1951 & - \\
$V_{2}$ & 806 & 1211 & 2500 & - & 469 & $\mathbf{8 6 9}$ & 2357 & - \\
$V_{\text {c1 }}$ & 51 & 50 & 50 & - & 56 & $\mathbf{5 0}$ & 82 & - \\
$V_{c 2}$ & 51 & 50 & 51 & - & 51 & $\mathbf{5 0}$ & 51 & - \\
$C V_{\text {setpoint }}$ & 484 & 495 & 439 & - & 489 & $\mathbf{4 9 5}$ & 441 & - \\
\hline
\end{tabular}

$\chi_{p}$ represents the structure of the cooling system: $\chi_{p}=0$ counter-current and $\chi_{p}=1$ co-current. $\chi_{c v}$ represents the structure of controlled variables: $\chi_{c v}=0$, i.e., $T_{2}$ is $\mathrm{CV}$ and $\chi_{c v}=1$, i.e., $T_{1}$ is CV. $\chi_{m v}$ represents the structure of manipulated variables: $\chi_{m v}=0$, i.e., $T_{f}$ is MV and $\chi_{m v}=1$, i.e., $Q_{c}$ is MV. (1) Inversion of the process is infeasible (See Figs. 10a, b.).

(2) The maximum allowable temperature of the cooling water leaving the process is violated. 

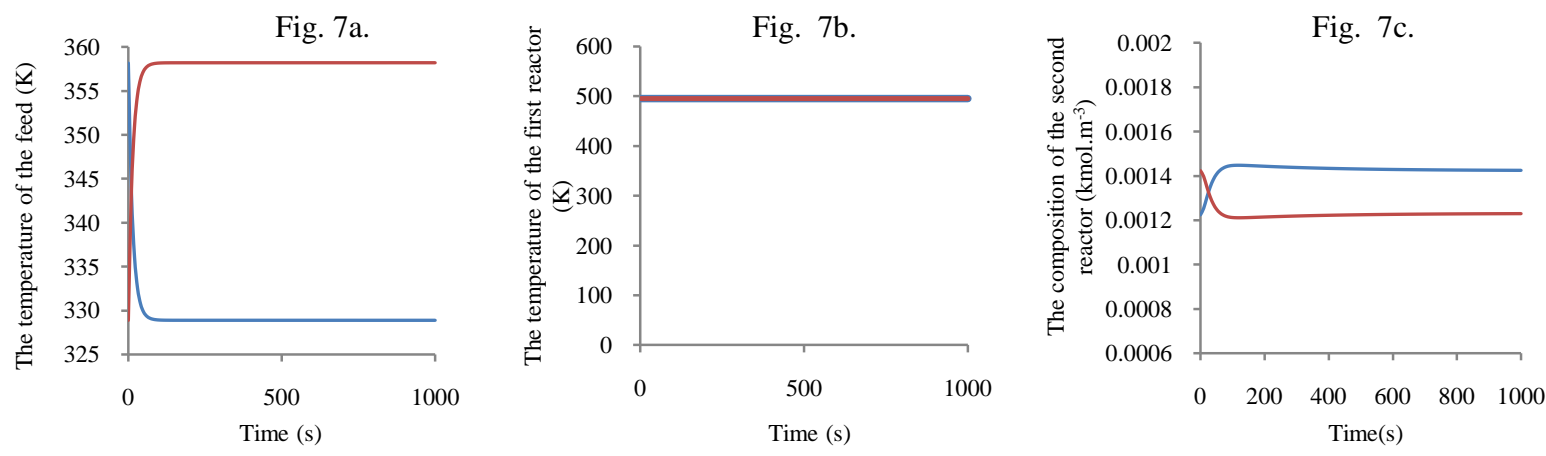

Figs. 7. Results for two disturbances. Trajectories of a) the feed temperature as the manipulated variable, b) the temperature of the first reactor as the controlled variable (overlaid on each other), c) the composition in the second reactor

Fig. 8a.

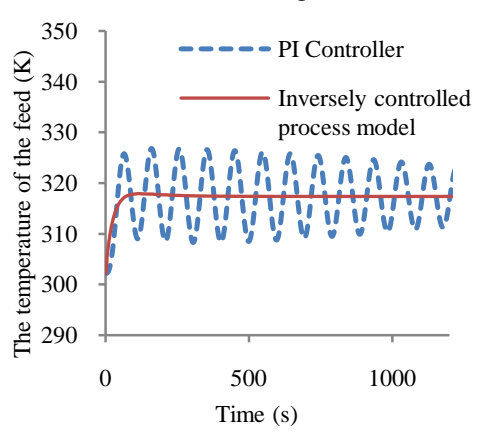

Fig. 8b.

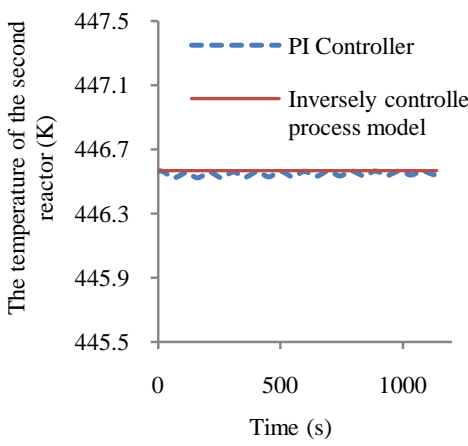

Fig. 8c.

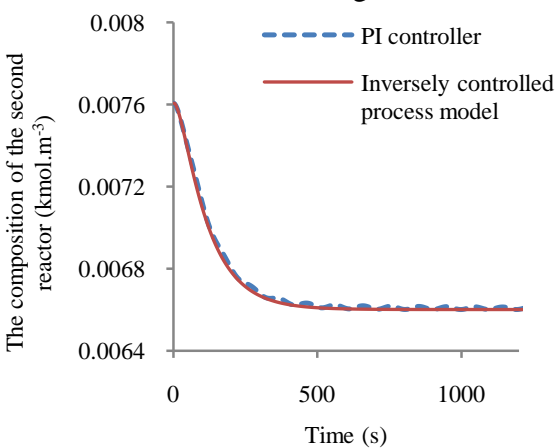

Figs 8. Comparison between the results of the conventional method (from [12]) and the inversely controlled process model for the same disturbance. Trajectories of a) the feed temperature as the manipulated variable, b) the temperature of the second reactor as the controlled variable (overlaid on each other), c) the composition in the second reactor

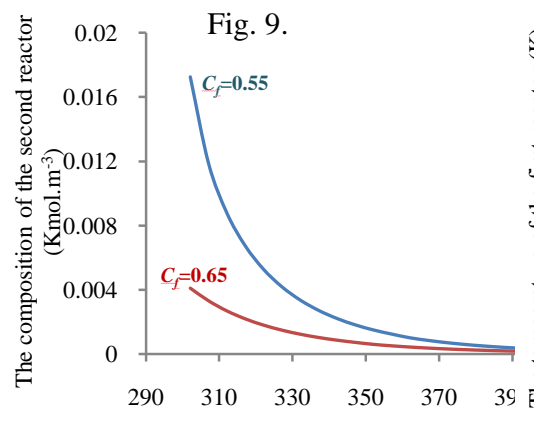

The temperature of the feed (K)

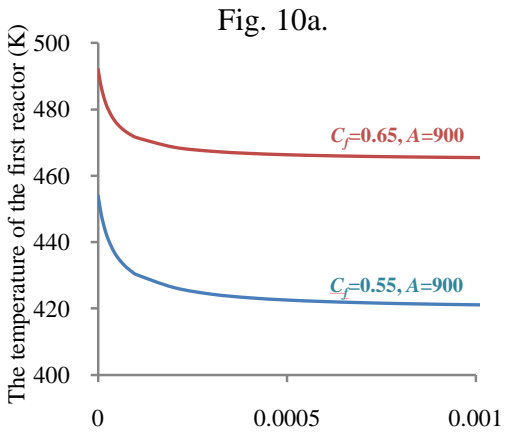

The flowrate of the cooling water $\left(\mathrm{m}^{3} \cdot \mathrm{s}^{-1}\right)$

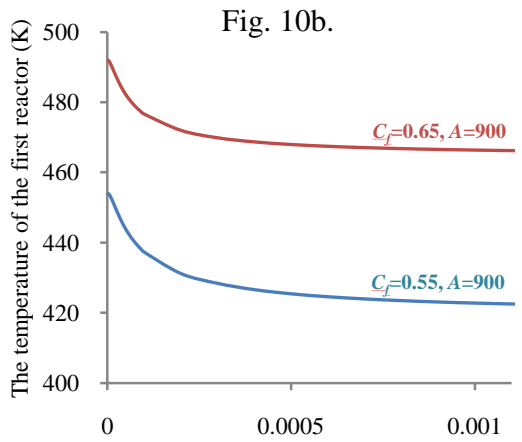

The flowrate of the cooling water $\left(\mathrm{m}^{3} \cdot \mathrm{s}^{-1}\right)$
Fig. 9. The variation of the composition of the second reactor with the feed temperature for the co-current structure
Figs. 10. The variation of the temperature of the first reactor with the flowrate of the cooling water, for a) the co-current structure, b) the counter-current structure. 


\section{Discussion}

In this section, firstly the results of the novel co-design optimization are explained in Section 6.1. and compared with the results of the conventional optimization from [12], in Section 6.2.. The aim is to establish the advantages of the proposed method over the conventional one. The cases in which the inversion of the process model was not possible (the blank columns in Table 2) are explained and justified in Section 6.3.

\subsection{The results of the proposed optimization framework}

The best process and control structure, selected by the proposed optimization framework, is structure 6. in which the temperature of the first reactor $T_{1}$ is the controlled variable and the feed temperature $T_{f}$ is the manipulated variable. The process structure is co-current heat transfer.

The optimal trajectories of the feed temperature are shown in Fig. 7a. They show a fast and smooth response. The optimal trajectories of the temperature of the first reactor as the controlled variable are shown in Figs 7b. These temperature trajectories are two straight lines which overlaid on each other and are not distinguishable. The optimal trajectories of the composition of the second reactor are shown in Fig 7c. Features of interest are high conversion, and very small changes caused by disturbances, as shown by the small scale of vertical axis in Fig. 7c.

A comparison between different structures reveals that the feed temperature is a more effective manipulated variable than the flowrate of the cooling water which results in infeasible solutions. In addition, in most structures the volumes of the cooling jackets are at their lower bounds because the heat exchanger hold-up reduces the dynamic performance. The setpoints of the controlled variables in most cases (not structures 3, 7, where the constraints are active) are increased from the base case design in order to make the process insensitive to disturbances. This is explained in the next section. The case study involves both heat transfer and heat generation. The selected structure has the feature that extra heat generated by disturbances in the feed composition leaves the process immediately in the co-current structure. However, in a counter-current structure the extra heat is returned back to the process by the cooling water and is circulated in the process before it completely diminishes.

\subsection{Comparison between the results of the novel and conventional optimization frameworks}

Flores-Tlacuahuac, et al [12] reported that their optimizer selected the temperature of the first reactor as the controlled variable and the feed temperature as the manipulated variable. They reported the counter-current heat exchange as the optimal process structure in the fifth and sixth parts of their results. Those parts of their results represented the results of a conventional co-design optimization.

In addition, Flores-Tlacuahuac, et al [12] reported that controlled variables remains constant within $0.02-0.1^{\circ} \mathrm{C}$ from the desired setpoint. By contrast, in the new optimization framework the controlled variable is maintained exactly at its setpoint because of the perfect control strategy.

Selection of the temperature of the first reactor as the controlled variable is going to inferentially control the composition of the reactant in the second reactor, which represents the conversion extent. When the process faces the disturbances, maintaining the controlled variable at its setpoint must suppress the variation in the composition of the second reactor. A good measure of success of the control structure is the variation of the composition of the second reactor due to disturbances. In the conventional optimization framework, for a change of $0.05 \mathrm{kmol} . \mathrm{m}^{-3}$ in the feed composition, the composition of the second reactor varies in the range of $0.002 \mathrm{kmol}^{-3} \mathrm{~m}^{-3}$ (Fig. 10 of [12]). The variation in the product composition is $4 \%$ of the variation in the feed composition. However, in the co-design optimization framework (Fig 4), for a change of $0.1 \mathrm{kmol} . \mathrm{m}^{-3}$ in the feed composition, the composition of the second reactor varies by $0.0002 \mathrm{kmol} . \mathrm{m}^{-3}$. The attenuation of the disturbance is about twenty times greater than with the conventional method. The reason for the success of the novel co-design framework is not due to the perfect control assumption but is because the new optimization 
framework was successful in recognizing the effects of the nominal temperature of the feed, shown in Fig. 9.

Fig. 9. shows the variation of the composition of the second reactor with the feed temperature. The top profile is when the feed composition is $C_{f}=0.55 \mathrm{kmol} . \mathrm{m}^{-3}$ and the bottom profile is when the feed composition is $C_{f}=0.65 \mathrm{kmol} . \mathrm{m}^{-3}$. Other process variables are at their nominal values (Table 1 ). The area between these two profiles is the operating region when disturbances occur. This figure reveals that by increasing the feed temperature the composition of the second reactor becomes insensitive to the disturbance in the feed composition, resulting in a tighter control. Similar analysis shows the same results for other structures also.

These results are achieved because the objective function (Eqn. 19) in the new co-design framework is process-oriented. The difference compared to reference [12] is that there is no term in the objective function for controller performance because of the perfect control assumption. In short, the perfect control approach to co-design finds the best combination of controlled and manipulated variables for optimizing process economics and the costs of control (manipulated variable movements and rates of change).

The point is illustrated in Figures 8a-c which compare the closed loop performance with the optimized PI controller from reference [12] and the perfect control using dynamic inversion of the same model with the controlled variables held at their set points according to Eqn. (1). The plots show the trajectories of the controlled variable $T_{2}$, the manipulated variable $T_{f}$, and the composition of the second reactor $C_{2}$. In each figure, two trajectories are illustrated. The first trajectory with oscillatory behaviour is reconstructed by simulation using the reported controller parameters, $K_{p}=500$ and $K_{I}=0.357 \mathrm{~s}$ from Table 5 of [12]. The time trajectories are the same as the time trajectories in Fig. 5 . of [12] and are shown using the dashed curves. The second trajectory with smooth behaviour is generated by the inversely controlled process model.

The implication of the smooth behaviour of the inversely controlled process model compared to the oscillatory behaviour of the optimal PI controller is that the oscillatory response must be attributed to the controller rather than the process. It suggests the type of controller assumed in the co-design was too restrictive, thus illustrating that parameterzing the controller type and tuning may not be beneficial.

Finally, as well as producing a well optimized co-design, the new co-design approach has achieved a reduction in the complexity of the problem because the differential and algebraic equations of the controller model are replaced by a set of explicit algebraic equations. Thus Eqns. (11-14) are replaced by Eqns. $(17,18)$ which reduces the number of equations and state variables. In addition, the tuning parameters of controllers will increase the number of optimization variables (e.g., from 8 to 10 in the small example of this article), which in large-scale industrial problems can even render the problem intractable.

\subsection{Uncontrollable process structures}

During the optimization solving, two uncontrollable structures were detected. In those structures, the flow rate of the cooling water was the manipulated variable and the temperature of the first reactor was the controlled variable. These uncontrollability issues manifested themselves as the failure of the integrator of the Aspen Custom Modeler (ACM) solver due to violation of bounds on the variables. Sensitivity studies using a steady-state model gave insights into the failures.

Fig. 9a. shows two steady-state analyses which demonstrate the variation of the temperature of the first reactor with the flowrate of the cooling water. The cooling water flows in a co-current structure. One profile is calculated for $C_{f}=0.55 \mathrm{kmol} . \mathrm{m}^{-3}$, and the other profile is for $C_{f}=0.65 \mathrm{kmol} . \mathrm{m}^{-3}$. Other process variables are at their nominal values (Table 1). If the process has state controllability then a horizontal tie-line must exists that connects the two profiles. Unfortunately, such a temperature does not exist and the process inversion is not possible. Similar results are shown in Fig. 9.b. for the 
counter-current structure in which the temperature of the first reactor is the controlled variable and the flowrate of the cooling water is the manipulated variable.

\section{Conclusion}

In this paper, a novel optimization framework for co-design is presented which disentangles the complexities associated with controllers by using the assumption of perfect control. In this framework instead of a combined model which includes models of the controllers, the inverse of process model is used. The treatment is based on the notion of functional controllability in which the process inputs (the required values of manipulated variables) are generated from the process outputs (the desired value of controlled variables) by inversion of the dynamic process model.

Since the design strongly depends on the values of disturbances, the proposed co-design framework falls in the category of optimization under uncertainty. Therefore, the mathematical formulation of the optimization problem is developed as a stochastic mixed integer dynamic optimization problem. The article also suggested a solving methodology based on an NLP solver linked with an embedded DAE solver for sequential integration.

The proposed methodology was benchmarked on a case study of two series reactors, which was previously used by [12] . The results demonstrated the advantage of the proposed framework over the conventional one due to disentangling the complexities of the controllers from the presentation of the co-design problem.

\section{Acknowledgement}

Mahdi Sharifzadeh gratefully acknowledges partial financial support from the 2010 and 2011 ISA Educational Foundation scholarships, the Burkett Scholarship and Ure bursary award of the Department of Chemical Engineering and Chemical Technology, Imperial College London. Nina F. Thornhill gratefully acknowledges the sponsorship of ABB and the Royal Academy of Engineering for the Research Chair in Process Automation at Imperial College London.

\section{References}

[1] V. Sakizlis, K.Vakamudi, A. Coward, I. Mermans, Advanced process control in the plant engineering and construction phases. Hydrocarbon Processing, October 2010.

[2] W. L. Luyben, Chapter A1: The need for simultaneous design education, in: P. Seferlis, C. M. Georgiadis (Eds), The Integration of Process Design and Control, Elsevier Science B.V. Amsterdam, 17 (2004) pp. 10-41.

[3] P. Seferlis, M. C. Georgiadis, The Integration of Process Design and Control, Elsevier Science B.V., Amsterdam, 2004.

[4] V. Sakizlis, J. D. Perkins, E. N. Pistikopoulos, Recent advances in optimization-based simultaneous process and control design, Comput. Chem. Eng. 2004, 28 (10) 2069- 2086.

[5] R. Grosch, M. Mönnigmann, W. Marquardt, Integrated design and control for robust performance: Application to an MSMPR crystallizer, J. Process Control, 18 (2) (2008) 173-188.

[6] O. J. Rojas, J. Bao, P. L. Lee, A dynamic operability analysis approach for nonlinear processes, J. Process Control, 17(2) (2007) 157-172.

[7] L.A. Ricardez-Sandoval, H.M. Budman, P.L. Douglas, Simultaneous design and control of processes under uncertainty: A robust modelling approach, J of Process Control, 18 ( 7-8) (2008) 735752. 
[8] L.A. Ricardez-Sandoval, H.M. Budman, P.L. Douglas, Simultaneous design and control of chemical processes with application to the Tennessee Eastman process, J. Process Control, 19(8) (2009) 1377-1391.

[9] K.-U. Klatt, W. Marquardt, Perspectives for process systems engineering-Personal views from academia and industry. Comput. Chem. Eng., 33 (2009) 536-550.

[10] J. Moon, S. Kim, A. A. Linninger, Integrated design and control under uncertainty: embedded control optimization for plantwide processes, Comput. Chem. Eng., In Press, Accepted Manuscript. doi:10.1016/j.compchemeng.2011.02.016.

[11] M. Sharifzadeh, N.F. Thornhill, Optimal controlled variable selection using a nonlinear simulation-optimization framework, ESCAPE 2011, May28-June1, Porto Carras Greece.

[12] A. Flores-Tlacuahuac, L. T. Biegler., Simultaneous mixed-integer dynamic optimization for integrated design and control, Comput. Chem. Eng., 31(5-6) (2007) 588-600.

[13] S. J. Qin, T. A. Badgwell, A survey of industrial model predictive control technology, Control Engineering Practice, 11(7) (2003) 733-764.

[14] L. T. Biegler, Nonlinear programming: concepts, algorithms, and applications to chemical processes, Society for Industrial \& Applied Mathematics, MOS-SIAM series on optimization, Philadelphia, PA, 2010.

[15] I. K. Kookos, J. D. Perkins, An algorithmic method for the selection of multivariable process control structures, J. Process Control, 12(1) (2002) 85-99.

[16] Z. Yuan, B. Chen, J. Zhao, An overview on controllability analysis of chemical processes, AIChE J., 57(5) (2011) 1185-1201.

[17] M. Morari, E. Zafiriou. Robust Process Control, Prentice-Hall International, Englewood Cliffs, New Jersey., 1989.

[18] S. Skogestad, I. Postlethwaite, Multivariable Feedback Control: Analysis and Design, 2nd ed., John Wiley \& Sons, Chichester, 2005.

[19] L. W. Russell, J. D. Perkins, Towards a method for diagnosis of controllability and operability problems in chemical plants, Chem. Eng. Res. Des., 65 (1987) 453-461.

[20] L. T. Biegler', A. M. Cervantes, A. Wächter, Advances in simultaneous strategies for dynamic process optimization, Chem. Eng. Sci., 57 (4) (2000) 575-593.

[21] M. Jelali, An overview of control performance assessment technology and industrial applications, Control Engineering Practice, 14( 5), (2006) 441-466.

[22] S.J. Qin, Control performance monitoring - A review and assessment, Comput. and Chem. Eng., 23 (1998) 178-186.

[23] V. N. Sahinidis, Optimization under uncertainty: state-of-the-art and opportunities. Computers \& Chemical Engineering. 28(6-7) (2004) 971-983.

[24] T. J. McAvoy, Synthesis of plantwide control systems using optimization, Ind. Eng. Chem. Res., 38 (8) (1999) 2984-2994.

[25] H. H. Rosenbrock, State-space and Multivariable Theory, Nelson, London, 1970. 
[26] J. A. Caballero, A. Odjo, I. E. Grossmann, Flowsheet optimization with complex cost and size functions using process simulators. AIChE J., 53 (9) (2007) 2351-2366. 